\title{
Modified Prone Positioning during Neurosurgery: Sphinx and Concorde Positions Revisited
}

\author{
Deep Sengupta ${ }^{1}$ Surya K. Dube ${ }^{1,}$ Vanitha Rajago
${ }^{1}$ Department of Neuroanaesthesiology and Critical Care, All India \\ Institute of Medical Sciences, New Delhi, India
}

J Neuroanaesthesiol Crit Care 2021;8:123-125.

Neurosurgical procedures involve various patient positions such as supine, prone, lateral, and sitting position. Prone positioning is routinely used in neurosurgical practice to obtain access to posterior neuroanatomical structures like the posterior fossa or suboccipital region. ${ }^{1}$ Sphinx and Concorde positions are modifications of the conventional prone position that are practiced less commonly in neurosurgery, probably because it requires neuroanesthesiologists and neurosurgeons, who are familiar with these positions, and more manpower, skills, and expertise to achieve the final positioning. Since these two positions are less commonly encountered in neuroanesthesiology practice, reports about these two positions in neuroanesthesiology literature are scarce. So, we have tried to review the indications, positioning method in the operating room, advantages, and disadvantages of these modified prone positions, with further modifications added to the classical methods described earlier to further facilitate the surgical practice and perioperative care.

\section{Sphinx Position}

The "sphinx" or "sea-lion" position was first devised by Suzuki et al in 1983 for total removal of a falcotentorial region meningioma by biparieto-occipital craniotomy, wherein they positioned the patient prone with hyperextended neck with $20^{\circ}$ elevation of upper and lower halves of the body. ${ }^{2}$ Since then, this position has undergone evolution and has been used in a multitude of surgeries requiring exposure to vertex and posterior skull regions, in order to achieve access to the anterior and posterior aspects of the cranial vault for reconstruction in cases of craniosynostosis. ${ }^{3}$ Nonetheless, the advent of new positioning techniques like the gel-filled collar technique, which focused on anterior portion of the cranial vault, and the angled horseshoe technique, which focused on the posterior portion, have decreased sphinx positioning usage, except in cases where superior access to the anterior and posterior aspects of the cranial vault is desired in a single setting. ${ }^{4}$

Published online August 10, 2020
DOI https://doi.org/

10.1055/s-0040-1715356

ISSN 2348-0548.
Address for correspondence Surya K. Dube, MD, DM, Department of Neuroanaesthesiology and Critical Care, 7th Floor, Neurosciences Center, All India Institute of Medical Sciences, New Delhi 110029, India (e-mail: surya.dube@yahoo.co.in).

\section{Indications $\mathbf{2}^{2,5,6}$}

Sphinx position is indicated in total or complex calvarial reconstruction, strip craniectomy, and for lesions in the falcotentorial region.

\section{Positioning ${ }^{7}$}

The patient is positioned in a prone position with the head supported at the malar area. In general, a horseshoe headrest is utilized. Reverse Trendelenburg position (at 30 to 45 degrees) is employed to prevent cervical hyperextension. Anterior and posterior parts of the skull can be accessed by tilting of the table. Arms are padded and the torso is stabilized by applying harnesses or strapping at the thighs or gluteal region (-Fig. 1). Careful securing of the tracheal tube prevents inadvertent displacement during the surgery. After the final positioning is achieved, the breath sounds must be checked bilaterally. Periodic reassessment of the positioning is important, and it must be done intermittently throughout the surgery.

\section{Advantages $^{7}$}

This position provides maximum accessibility to the cranial vault, especially in patients with scaphocephaly in terms of both anterior and posterior approaches. It bestows neurosurgeons with adequate access to posterior cerebral structures and also facilitates venous return resulting in a better surgical field. The tilting of table during the procedure to further maximize the access is possible with minimal effect on the initial positioning, which is important for neuroanesthesiologists. It also provides adequate depth and wide visualization of the operative field in lesions pertaining to the falcotentorial region with minimal retraction of brain parenchyma. $^{2}$

\section{Disadvantages $\mathrm{s}^{3,8}$}

This position entails a high-risk of venous air embolism due to a multitude of risk factors, including a surgical site above the right atrium, a large head of neonates and infants relative

(c) 2020. Indian Society of Neuroanaesthesiology and Critical Care.

This is an open access article published by Thieme under the terms of the Creative Commons Attribution-NonDerivative-NonCommercial-License, permitting copying and reproduction so long as the original work is given appropriate credit. Contents may not be used for commercial purposes, or adapted, remixed, transformed or built upon. (https://creativecommons.org/licenses/by-nc-nd/4.0/).

Thieme Medical and Scientific Publishers Pvt. Ltd. A-12, 2nd Floor, Sector 2, Noida-201301 UP, India 


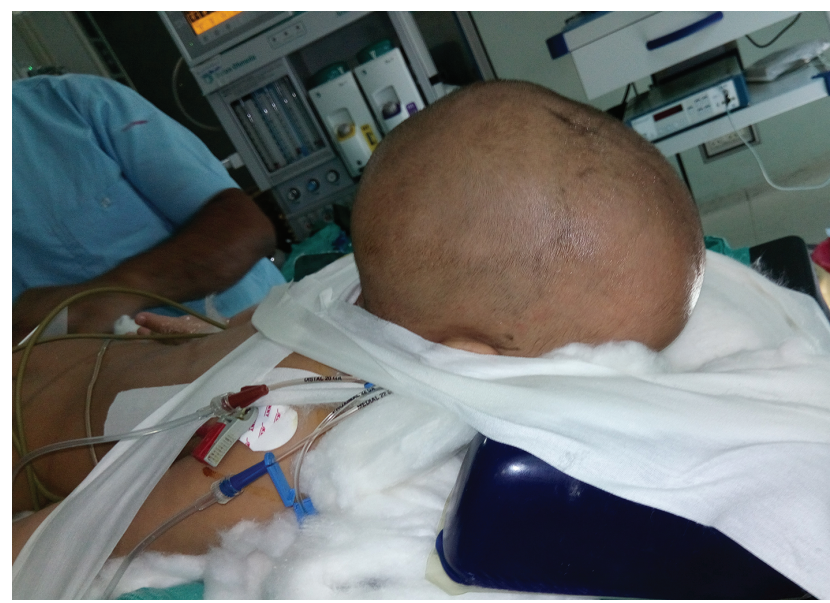

Fig. 1 Sphinx position

to adults, and increased blood loss in these surgeries, leading to a decrease in central venous pressure. There is also a possibility of inadvertent extubation during positioning and neck extension, which leads to a significant cephalad movement of the endotracheal tube tip. Since this position involves hyperextension, it is contraindicated in cases of cervical spine abnormality. ${ }^{2}$

\section{Concorde Position}

It was first described by Kobayashi et al for the infratentorial supracerebellar approach, and since then, it is being used for operating in midline posterior fossa region for pineal and cerebellar lesions. ${ }^{9}$ Concorde position is still preferred over sitting position by some neurosurgeons because of several complications associated with the latter like venous air embolism, airway edema, central cord syndrome, macroglossia, spinal cord myelopathy, and postoperative tension pneumocephalus. ${ }^{10}$ Moreover, positioning of the patient is relatively easier in the Concorde position than in the sitting position. ${ }^{10}$ The proponents of sitting position cite the surgical advantages like superior visualization of the intended area and improved preservation of lower cranial nerves. ${ }^{11,12}$

\section{Indications $\mathbf{s}^{1,9}$}

This position is used for a surgical approach to the occipital transtentorial and supracerebellar infratentorial regions of posterior fossa.

\section{Positioning 1,9,13,14}

Concorde positioning is achieved by placing the patient in a reversed Trendelenburg position and as far as possible towards the left edge of the table. The patients head is fixed with a $15^{\circ}$ right-side tilting. The arms are tucked and wrapped along the sides of the patient with adequate cushioning, and the trunk is stabilized by putting chest rolls underneath. Lower limbs are flexed at the knees with adequate padding. The patient is secured with a strap around the thighs or buttocks to prevent slippage toward the caudal end

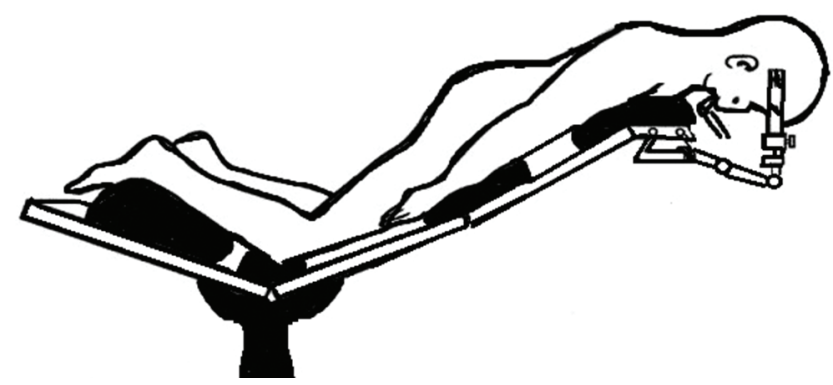

Fig. 2 Concorde position.

of the table. If the surgical site involves the cervical spine or suboccipital area, strapping from the shoulders toward the feet is desirable. Final positioning is achieved by alternately flexing the knees and progressively increasing the reversed Trendelenburg position. The patient overhangs at the cranial end of the table to achieve the required flexion of the head. The head is flexed maximally to achieve desired exposure of the posterior fossa, ensuring at least two finger breadth space between the chin and manubrium ( $\boldsymbol{- F i g}$. 2). Positioning the head at the level of the heart minimizes the risk of venous air embolism. The surgeon is usually seated on the patient's left side if the lesion is on the right side and on the right side for a left-sided lesion, which ensures natural position to operate. In this position, there is a possibility for interference during surgery by patient's shoulder which is nearest to the surgeon during visualization and instrumentation. This may not be completely avoided with the taping of shoulders and pulling it away from the cephalad region, especially in a muscular, short necked, or obese patients. Kyoshima et al proposed a modified arm down Concorde position to obviate the above issue by allowing the patient's arm to hang down by the surgeon's side. ${ }^{14}$ Other techniques proposed to tackle the shoulder issue were prone oblique position and asymmetrical fixation of the head by skew head rotation in the Sugita head holder. ${ }^{13,15}$ Once the final position is achieved, it is essential to check the endotracheal tube position by performing bilateral auscultation and ensure there is no compression of pressure points on the face and eyes.

\section{Advantages}

Concorde positioning facilitates the descent of the cerebellum due to its weight, which ensures minimal retraction on the cerebellum, adequate exposure of the desired region, and a greater degree of surgical maneuverability for the surgeon. ${ }^{9}$ Placing the patient to the maximum possible far edge of the table and tilting the head toward right keeps the midline of the patient's head axis straight in the surgical field for comfortable and accurate surgical manipulation. ${ }^{13}$

\section{Possible Complications/Disadvantages ${ }^{10,13,16}$}

This position can cause various postoperative problems like neck pain, pressure sore/necrosis, and brachial plexus injury secondary to excessive shoulder stretching. Besides this, probable cerebral venous outflow/lymphatic drainage obstruction may lead to swelling of the face, tongue, and neck and potential airway obstruction in the postoperative phase. 
Moreover, various authors have reported difficulty in obtaining adequate neck flexion, as the patient's shoulders exceeded the operating table edge and pin site misplacement due to part loading of the body weight on the three pin holders. Gulsen et al proposed a technique to overcome this issue, wherein they first placed the three pin holders in the supine position at an obtuse angle and then turned the patient prone, finally achieving the Concorde position. ${ }^{10}$ Rare but serious complications may include posterior circulation insufficiency (vertebrobasilar dissection/thrombosis) due to compression/kinking of the vertebral arteries between occipital condyles and C1 arch on account of hyperflexion. Excessive flexion may rarely lead to bilateral carotid compression.

\section{Conclusion}

The positioning of the patient for accessing the posterior fossa midline structures may require modified prone positions to maximize access to the desired surgical regions with satisfactory exposure and minimal retraction on the brain. Technical expertise, thorough planning, and adequate padding of dependent structures are of paramount importance for modified prone positioning. Knowledge regarding these positions will enable the neuroanesthesiologist to foresee and manage any inadvertent complications and take better care of the patient perioperatively. Constant vigilance of the neuroanesthesiologist and communication with the neurosurgeon is of vital importance throughout the procedure.

Acknowledgement

We would like to acknowledge Miss Rishima Dube for her help in preparing the artwork for the manuscript.

\section{Conflict of Interest}

None declared.

\section{References}

1 Rozet I, Vavilala MS. Risks and benefits of patient positioning during neurosurgical care. Anesthesiol Clin 2007;25(3):631-653
2 Suzuki M, Sobata E, Hatanaka M, Suzuki S, Iwabuchi T, Makiguchi K. Total removal of a falcotentorial junction meningioma by biparietooccipital craniotomy in the sea lion position: a case report. Neurosurgery 1984;15(5):710-714

3 Johnson JO, Jimenez DF, Tobias JD. Anaesthetic care during minimally invasive neurosurgical procedures in infants and children. Paediatr Anaesth 2002;12(6):478-488

4 Francel PC, Bell A, Jane JA. Operative positioning for patients undergoing repair of craniosynostosis. Neurosurgery 1994;35(2):304-306

5 Stricker PA, Fiadjoe JE. Anesthesia for craniofacial surgery in infancy. Anesthesiol Clin 2014;32(1):215-235

6 Berry-Candelario J, Ridgway EB, Grondin RT, Rogers GF, Proctor MR. Endoscope-assisted strip craniectomy and postoperative helmet therapy for treatment of craniosynostosis. Neurosurg Focus 2011;31(2):E5

7 Mayorga-Buiza M, Tosca M, Rivero-Garvia MM-RJ. 9th International Update on Neuroanesthesia and Neurointensive Care EURONEURO 2016: Barcelona, Spain, April 14-16, 2016. J Neurosurg Anesthesiol 2016;28(2):S1-S51

8 Sugiyama K, Yokoyama K. Displacement of the endotracheal tube caused by change of head position in pediatric anesthesia: evaluation by fiberoptic bronchoscopy. Anesth Analg 1996;82(2):251-253

9 Kobayashi S, Sugita K, Tanaka Y, Kyoshima K. Infratentorial approach to the pineal region in the prone position: Concorde position. Technical note. J Neurosurg 1983;58(1):141-143

10 Gulsen S, Yilmaz C, Ozdemir O, Gerilmez A, Caner H, Altinors N. Placement of three-pin head holders in the Concorde position. Turk Neurosurg 2010;20(2):136-141

11 Charbel F, Kehrli P, Pain L. [The sitting position in neurosurgery: the viewpoint of the surgeon]. Ann Fr Anesth Reanim 1998;17(2):160-163

12 Gale T, Leslie K. Anaesthesia for neurosurgery in the sitting position. J Clin Neurosci 2004;11(7):693-696

13 Takasuna H, Tanaka Y. The modified concorde position with an intraoperative skew head rotation: Technical note. Neurol Med Chir (Tokyo) 2015;55(8):680-682

14 Kyoshima K. Arm-down Concorde position: a technical note. Surg Neurol 2002;57(6):443-445

15 Kikuta KI, Miyamoto S, Kataoka H, Satow T, Yamada K, Hashimoto $\mathrm{N}$. Use of the prone oblique position in surgery for posterior fossa lesions. Acta Neurochir (Wien) 2004;146(10):1119-1124

16 Kumar A, Bhattacharjee S, Sahu BP, Kulkarni D.. Brain death after Concorde positioning for supracerebellar-infratentorial approach: Unanswered questions and lessons learned. Surg Neurol Int 2012;3-37 